\title{
Análise Espaço-Temporal da Mobilidade Urbana de Diferentes Grupos Socioeconômicos: Uma Abordagem Multicamadas
}

\author{
Fernanda R. Gubert ${ }^{1}$, Priscila Santin ${ }^{1}$, \\ Anelise Munaretto ${ }^{1}$, Mauro Fonseca ${ }^{1}$, Thiago H. Silva ${ }^{1,2}$ \\ ${ }^{1}$ Universidade Tecnológica Federal do Paraná (UTFPR) \\ Curitiba, Brasil \\ ${ }^{2}$ University of Toronto \\ Toronto, Canada \\ fernandagubert, priscila@alunos.utfpr.edu.br
}

\begin{abstract}
Resumo. Este trabalho analisa a mobilidade urbana de Curitiba por meio do uso do transporte público e com a população dividida em estratos socioeconômicos. Para estudar o grafo de mobilidade estabelecido e seus padrões espaço-temporais foi aplicada uma abordagem multicamadas denominada Multi-Aspect Graph (MAG) com quatro aspectos. Os resultados detalham padrões que não podem ser observados em um grafo tradicional, tais como os deslocamentos mais frequentes bem como os pontos mais centrais da cidade levando em consideração todos os aspectos modelados. A modelagem utilizada possibilita um entendimento estratégico do uso do sistema público de transporte benéfico para diferentes aplicações, tais como atuações em situações de emergência, como surtos de doenças de rápido contágio.
\end{abstract}

\section{Introdução}

O debate sobre mobilidade urbana no Brasil tem sido um tema muito discutido entre os administradores públicos das grandes cidades [Prefeitura Municipal de Curitiba 2020a, Prefeitura Municipal de Porto Alegre 2019, Governo de São Paulo 2019]. A mobilidade urbana refere-se à facilidade de movimentação da população e bens no espaço geográfico das cidades, constituindo um componente da qualidade de vida aspirada por seus habitantes [Araujo et al. 2011]. Um possível modelo para representar a mobilidade urbana é através de grafos, que consiste em mapear os elementos do sistema em vértices e arestas. A utilização de grafos no estudo da mobilidade auxilia na codificação das informações sobre as diversas interações entre as partes, criando um modelo que facilita a compreensão do comportamento coletivo [Silva et al. 2019].

A dinâmica da mobilidade urbana pode ser estudada sob vários aspectos, como tempo e espaço, por exemplo, e para que todas suas nuances possam ser analisadas simultaneamente faz-se necessário o uso de um recurso de multicamadas ao invés de um grafo tradicional. Tal estrutura é conhecida como um Grafo Multi Aspecto (Multi-Aspect Graph (MAG)), sendo uma extensão da teoria de grafo tradicional que permite analisar a interação entre as camadas de um sistema complexo. Apesar de tais estudos datarem de décadas, os grafos multicamadas tornaram-se uma das direções recentes mais importantes na área de ciência de redes [Kivela et al. 2014].

Este trabalho tem como objetivo utilizar um MAG para caracterizar um sistema complexo, neste caso, a mobilidade urbana usando transporte público na cidade de $\mathrm{Cu}$ ritiba. Nesse sentido, faz-se uma comparação com os resultados apresentados em nosso trabalho prévio [Santin et al. 2020], no qual criamos um modelo representando os deslocamentos de origem e destino dos usuários do transporte público de Curitiba utilizando 
a estrutura de um grafo tradicional, e a partir desta estrutura extraímos padrões espaçotemporais considerando subconjuntos correspondentes a estratos socioeconômicos.

Os resultados evidenciam, por exemplo, as movimentações por horário e classe socioeconômica mais frequentes, além da identificação de pontos centrais da cidade levando em consideração simultaneamente os aspectos modelados. Observa-se que a não consideração de aspectos como classe socioeconômica ou tempo representam compreensões parciais do fenômeno em estudo, que podem levar a conclusões equivocadas. Os resultados deste trabalho sugerem ainda que o modelo proposto provê um entendimento importante da mobilidade usando o transporte público que pode ser benéfico em diferentes aplicações, por exemplo, em intervenções em situações de emergência, como a pandemia gerada pelo COVID-19.

O restante do estudo está organizado da seguinte maneira. A Seção 2 apresenta os estudos relacionados. A Seção 3 mostra algumas informações relevantes sobre a cidade de estudo. Na Seção 4, apresentamos a metodologia aplicada neste trabalho. A Seção 5 discute os resultados. Finalmente, a Seção 6 apresenta a conclusão deste estudo.

\section{Trabalhos Relacionados}

Dado que a mobilidade urbana é uma parte fundamental do funcionamento de cidades, muitos estudos recentes focam no entendimento desse fenômeno de diferentes perspectivas, desde a mobilidade de grupos [Nunes et al. 2017] e de taxis [Martins and Cunha 2018], até a mobilidade considerando o transporte público de várias maneiras [Morais and Camargo 2019, Herek et al. 2018, Átila M. Silva Jr et al. 2018]. Boa parte dos trabalhos que visam entender padrões de mobilidade urbana representam esse fenômeno por meio de grafos [Silva et al. 2019]. Nesse sentido, os grafos com variação espaço-temporais se apresentam como um modelo útil para o estudo de redes com uma certa dinâmica, embora nem sempre esses dois atributos sejam explorados ao mesmo tempo.

Numa aplicação para o entendimento da mobilidade utilizando-se de uma representação espaço-temporal, Marques-Neto et al. [Marques-Neto et al. 2018] utilizaram-se de registros celulares para analisar a dinâmica espaço-temporal dos padrões de movimento dos participantes de eventos de grande escala. Baseando-se em três tipos diferentes de eventos: uma grande partida de futebol, um show de rock e uma celebração de Ano Novo, os autores foram capazes de melhorar a compreensão da mobilidade humana causada por esses eventos e desenvolveram um aplicativo para ajudar as operadoras de telefonia celular a planejar sua infraestrutura em grandes eventos.

Aplicando duas abordagens de clusterização (agrupamento) aos dados de cartão inteligente do transporte público da região metropolitana de Rennes na França, Mahrsi et al. [El Mahrsi et al. 2017] puderam extrair padrões de mobilidade temporal em dois pontos de vista, um em relação às estações e outro focado nos passageiros, considerando também o tipo de tarifa utilizado. Dentre seus resultados, foi possível identificar diferentes funções de uma estação, como estações residenciais usadas principalmente pela manhã, quando os passageiros estão indo ao trabalho, e estações de trabalho usadas apenas à noite, denotando o retorno dos passageiros às suas casas, assim como inferir o comportamento dos usuários, mostrando uma utilização esporádica do transporte por alguns ou práticas típicas de deslocamento. 
Utilizando-se também de cartões inteligentes para o estudo de mobilidade, Zhang et al. [Zhang et al. 2018] propõem uma estrutura analítica para comparar os padrões de mobilidade urbana extraídos de um conjunto de dados de cartões inteligentes de trânsito (ônibus e metrô) e um conjunto de dados de trajetórias de táxi por meio de GPS. Usando Cingapura neste estudo de caso e utilizando-se de matrizes de origem e destino extraídas de ambos os conjuntos de dados, os autores concluem que as distribuições espaciais extraídas da demanda de viagens de ambos os modos de transporte exibem altas correlações, mas com um grau maior de heterogeneidade no uso do espaço pelo transporte público. Os tempos de viagem decaem mais rápido para o uso do táxi, destacando a importância dos táxis para facilitar as viagens de longa distância. Além disso, cada um dos modos de transporte desempenham um papel específico na conexão de determinados lugares na cidade de Cingapura.

O trabalho de Rodrigues et al. [Rodrigues et al. 2018a] propõe uma metodologia para coletar e integrar dados de mobilidade urbana de fontes heterogêneas com o intuito de entender melhor a dinâmica das cidades. Os autores agregaram diferentes dados coletados das mídias sociais e do sistema de táxi da cidade de Nova York numa abordagem que utiliza a estrutura de um MAG para construir e avaliar uma ferramenta que permite entender a correlação espaço-temporal de dados urbanos de diferentes fontes.

Com uma abordagem de mobilidade em diferentes estratos socioeconômicos, $\mathrm{Xu}$ et al. [Xu et al. 2018] utilizam-se de informações de telefones celulares e propõem uma estrutura analítica para entender melhor os padrões de mobilidade humana nos diferentes estratos socioeconômicos da população. O mesmo acontece no estudo de Lotero et al. [Lotero et al. 2016], que analisa a mobilidade urbana entre duas cidades colombianas usando uma pesquisa de origem-destino e acoplando as informações de classificação socioeconômicas. No trabalho de [Rodrigues et al. 2018b], os autores analisaram a população do Rio de Janeiro com o objetivo de caracterizar a mobilidade urbana e encontrar padrões nos estratos sociais, a partir de dados de telefonia móvel. Foi encontrado que a média de ligações por dia decresce com o aumento da renda, assim como a distância média percorrida.

Em nosso estudo prévio [Santin et al. 2019] realizamos uma caracterização nas bases de dados de saúde e de transporte de Curitiba de forma a entender melhor as questões referentes à utilização desses sistemas e possibilitar uma adequada integração de dados. A empregabilidade de uma estrutura de dados do tipo Multi-Aspect Graph (MAG), não contemplada anteriormente, visa possibilitar uma análise mais abrangente e de forma integrada dos sistemas. Já em [Santin et al. 2020] estudamos a mobilidade urbana de Curitiba por meio do uso do transporte público da cidade. Os bairros da cidade foram utilizados para estabelecer o grafo de mobilidade, onde os vértices representavam os bairros e as arestas a existência de um deslocamento entre eles. Os usuários do transporte público foram divididos em subconjuntos correspondentes a estratos socioeconômicos e os padrões espaço-temporais foram explorados de maneira agregada naquele estudo. De acordo com os resultados, concluímos que com o aumento da riqueza a atividade matinal é adiada e a distribuição espacial das viagens torna-se mais localizada.

O presente estudo também analisa a mobilidade urbana nos diferentes estratos sociais, porém incorporando uma técnica não explorada nos trabalhos citados, a qual trata-se de uma abordagem que utiliza o Multi-Aspect Graph (MAG). Comparamos alguns resul- 
tados deste estudo com o apresentado anteriormente [Santin et al. 2020], identificando as nuances que o MAG permite em um estudo mais detalhado da mobilidade urbana de $\mathrm{Cu}$ ritiba. Por exemplo, a identificação dos horários em que ocorrem os deslocamentos mais intensos do grafo geral, com suas respectivas classes socioeconômicas, além dos locais de maior centralidade para essas classes, também com a indicação de horário.

\section{Descrição dos Dados}

Nesta seção são descritas brevemente informações relevantes sobre a cidade de Curitiba e como seu transporte público é estruturado. Além disso, informações sobre a estratificação social e econômica do Brasil são apresentadas.

\subsection{Curitiba e seu Transporte Público}

De acordo com o último censo demográfico do Brasil realizado pelo $\mathrm{IBGE}^{1}$, o município de Curitiba possui 1.751.907 habitantes em uma área total de $434.967 \mathrm{~km}^{2}$. A extensão Norte-Sul é de $35 \mathrm{~km}$ e a extensão Leste-Oeste é de $20 \mathrm{~km}$. Administrativamente, Curitiba é dividida em 10 Administrações Regionais, cobrindo seus 75 bairros. Todos esses bairros são atendidos pelo sistema de transporte público de Curitiba. Tal sistema de transporte possui corredores de transporte exclusivos, onde circulam ônibus biarticulados. Esses ônibus biarticulados passam por vários terminais de integração que recebem os ônibus alimentadores da vizinhança, permitindo a integração do sistema. O sistema também possui linhas circulares entre bairros, que permitem a passagem de um bairro para outro, sem ter que percorrer a região central da cidade, além de linhas diretas, que oferecem viagens mais rápidas com menos paradas no itinerário. Essas linhas diretas têm um tipo de ponto de ônibus específico para elas, assim como os terminais de integração. Outra característica importante do transporte público de Curitiba é a tarifa integrada. Pagando apenas uma passagem, o cidadão pode compor sua própria rota, movimentando-se pela cidade.

Desde 2002, Curitiba utiliza o sistema de bilhetagem eletrônica para o transporte público. A implementação desse sistema foi necessária para reduzir o fluxo de caixa em circulação no sistema de transporte, acelerar o embarque e a passagem de usuários pelas catracas, disciplinar e mensurar o uso do sistema de transporte por categorias que gozam de acesso e isenções gratuitos, além de reduzir os custos do sistema operacional [Taniguchi and Duarte 2012]. Segundo a URBS $^{2}$ [Urbanização de Curitiba S/A - URBS 2018] em 2018 uma média de 1.365.615 passageiros foram transportados a cada dia útil. Além disso, de acordo com o mesmo relatório, cerca de $60,96 \%$ das tarifas foram pagas usando o cartão transporte. O número de cartões transporte ativos em 2018 foi de 1.928.184, dividido em três das quatro categorias de transporte existentes, a saber: Cartão de Usuário, Cartão de Isenção, Cartão de Estudante e Cartão Único (para esta categoria, não há informações nos dados fornecidos pela URBS).

\subsection{Estratificação Social e Econômica no Brasil}

No Brasil, existem vários critérios disponíveis para classificar a sociedade de acordo com a renda familiar. No entanto, neste estudo, foi utilizado o critério da Secretaria de Assuntos Estratégicos (SAE) do governo federal, estabelecido em 2012 [Ninis et al. 2012].

\footnotetext{
${ }^{1}$ IBGE: Instituto Brasileiro de Geografia e Estatística.

${ }^{2}$ URBS (Urbanização de Curitiba S/A): empresa que gerencia o transporte público em Curitiba.
} 
A Tabela 1 apresenta o resumo dessas classes.

Tabela 1. Estratificação Socieconômica de acordo com a SAE

\begin{tabular}{|l|l|}
\hline \multicolumn{1}{|c|}{ Grupo } & \multicolumn{1}{c|}{ Renda Familiar } \\
\hline Classe 1 - Extremamente Pobre & até $\mathrm{R} \$ 324$ \\
\hline Classe 2 - Pobre & até $\mathrm{R} \$ 648$ \\
\hline Classe 3 - Vulnerável & até $\mathrm{R} \$ 1.164$ \\
\hline Classe 4 - Classe Média Baixa & até $\mathrm{R} \$ 1.764$ \\
\hline Classe 5 - Classe Média & até $\mathrm{R} \$ 2.564$ \\
\hline Classe 6 - Classe Média Alta & até $\mathrm{R} \$ 4.076$ \\
\hline Classe 7 - Classe Alta Baixa & até $\mathrm{R} \$ 9.920$ \\
\hline Classe 8 - Classe Alta & acima de $\mathrm{R} \$ 9.920$ \\
\hline
\end{tabular}

Essas informações de renda foram adquiridas por meio do Censo Demográfico Brasileiro de 2010 realizado pelo IBGE [Instituto Brasileiro de Geografia e Estatística - IBGE 2010]. Esta pesquisa nacional realizada pelo IBGE teve como objetivo retratar a população brasileira com suas características socioeconômicas e também estabelecer uma base para todo o planejamento público e privado para a década entre 2010-2020.

No Brasil, a classificação social de acordo com a renda familiar é dividida em classe alta, classe média e classe baixa. Com base nos estudos realizados pela SAE, esses grupos foram subdivididos, ficando a classe baixa em três grupos, a classe média também em três grupos e a classe alta em dois grupos. De acordo com essa classificação e a renda estimada por bairro, Curitiba apresenta apenas quatro das oito classes apresentadas na Tabela 1, a saber: Classe Média Baixa, Classe Média, Classe Média Alta e Classe Alta Baixa.

\section{Metodologia}

Nesta seção são descritos o conjunto de dados estudado e as etapas metodológicas mais importantes.

\subsection{Bases de Dados}

Para a análise da mobilidade urbana desenvolvida neste estudo, foram utilizados os dados fornecidos pela URBS. A base de transporte público está disponível para uso pelo público em geral, sem restrições. Para ter acesso a esse conjunto de dados, é necessário fazer uma solicitação à URBS. As informações contidas nessa base de dados são dos registros diários de cartões transporte e cada um desses registros apresentam 7 campos: Código da Linha, Nome da Linha, Código do Veículo, Número do Cartão, Data de Utilização, Data de Nascimento e Sexo. Além disso, esse conjunto de dados possui vários arquivos de informações complementares, como as linhas de ônibus existentes e as paradas de ônibus que as linhas atendem, com localização GPS e tabela horária do veículo. Alguns dados são estáticos e atualizados uma vez por dia. Outros dados, como a localização dos veículos, são atualizados praticamente em tempo real, pois os veículos enviam sua localização em média a cada 5 segundos. Todas essas informações são disponibilizadas por meio de arquivos .json.

Neste estudo, foram utilizados dois arquivos complementares relacionados ao transporte público de Curitiba: Veículos e Pontos de Paradas. O arquivo de Veículos contém a localização de todos os veículos que circularam em Curitiba. As informações 
contidas nesse arquivo são distribuídas em 5 campos: Código do Veículo, Latitude, Longitude, Data de Utilização e Código da Linha. Em seguida, o arquivo de Pontos de Paradas contém as informações de todos os pontos de ônibus existentes em Curitiba. Este arquivo possui nove campos de informações, a saber: Nome do Ponto, Número do Ponto, Latitude, Longitude, Sequência, Grupo, Direção, Tipo e Código da Linha.

\subsection{Processamento das Bases de Dados}

Para a criação do banco de dados foram utilizados 58 arquivos referentes as entradas de cartão transporte e 67 arquivos de dados sobre os veículos, totalizando 69 dias analisados (53 dias úteis e 16 dias de finais de semana). As entradas de cartão transporte representam no total 17.511.710 registros e os arquivos de veículos 129.461.200 registros.

Para inferir em qual bairro cada entrada de cartão foi realizada, procurou-se uma correspondência nos arquivos dos veículos usando o código da linha, código do veículo, data e hora aproximadas, obtendo desta forma a latitude e longitude de cada entrada de cartão. Cada par de latitude e longitude foi associado ao ponto de ônibus mais próximo da linha específica da entrada do cartão transporte. Este procedimento foi realizado usando uma estrutura de dados hierárquica, conhecida como Árvore- $R$. A Árvore- $R$ foi proposta por Antonin Guttman [Guttman 1984] e é amplamente usada como método de acesso ao espaço, permitindo a indexação de informações multidimensionais como coordenadas geográficas. No final desta etapa foi possível inferir o bairro para 5.388.638 registros de cartão transporte.

Para cada cartão transporte foi necessário associar um bairro de origem, que denominamos a moradia do usuário. Para isso, foram consideradas as entradas entre $5 \mathrm{~h}$ e 10h, pois esse é um período no qual as pessoas geralmente usam o transporte público para ir trabalhar ou estudar. Entretanto, era necessário identificar quantas entradas mínimas o cartão precisava ter registrado para que pudesse ser considerado. Traçando uma CDF com as entradas realizadas entre $5 \mathrm{~h}$ e $10 \mathrm{~h}$ e agrupando os dados por cartão, esse limiar foi definido em 10 entradas. Esse valor representa um bom compromisso entre o número de usuários filtrados e dados disponíveis para cada usuário, pois é possível representar aproximadamente $22 \%$ dos cartões, como pode ser visto na Figura 1, permitindo análises subsequentes com maior confiança. Após a exclusão das entradas dos cartões que apresentavam menos de 10 entradas, recuperou-se o bairro mais frequente no grupo de horário das $5 \mathrm{~h}$ às $10 \mathrm{~h}$ por cartão, obtendo 14.632 cartões transporte exclusivos com bairro de origem associado.

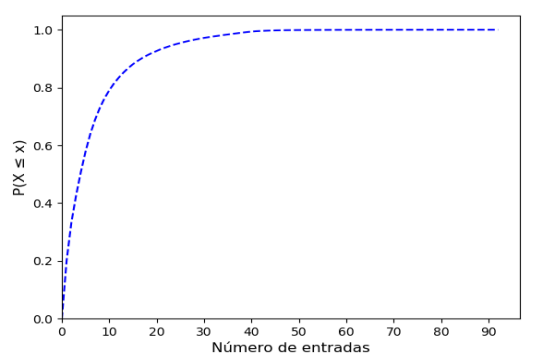

Figura 1. CDF das entradas de cartões realizadas entre $5 \mathrm{~h}$ e $10 \mathrm{~h}$ 


\subsection{Modelos e Métricas de Análise}

\subsubsection{Modelo Multicamadas}

Considerando um Grafo Multi Aspecto (Multi-Aspect Graph ou MAG) uma estrutura capaz de representar uma rede multicamadas variante no tempo, o modelo MAG proposto é dado por $H=(A, E)$, onde $E$ é o conjunto de arestas e $A$ a lista de aspectos que compõe o modelo. Cada aspecto $\alpha \epsilon A$ é um conjunto finito e o número de aspectos $p$ representa a ordem do MAG. Cada aresta $e \epsilon E$ é uma tupla com $2 \times p$ elementos. Todas as arestas possuem a forma $\left(a_{1}, \ldots, a_{p}, b_{1}, \ldots, b_{p}\right)$, onde $a_{1}, b_{1}$ são elementos do primeiro aspecto de $H, a_{2}, b_{2}$ são elementos do segundo aspecto de $H$ e assim por diante, até $a_{p}, b_{p}$, os quais são elementos do $p$-ésimo aspecto de $H$ [Wehmuth et al. 2016].

Para analisar a mobilidade do transporte público de Curitiba foi criado um MAG com ordem $p=4$, ou seja, 4 aspectos. Esses aspectos são: classe socioeconômica do usuário que está realizando o deslocamento, ou seja, a classe do bairro de moradia do usuário, sendo CMB (Classe Média Baixa), CM (Classe Média), CMA (Classe Média Alta) e CAB (Classe Alta Baixa); os bairros da cidade; 24 instantes de tempo (cada instante corresponde a $1 \mathrm{~h}$ do dia) e classe socioeconômica do bairro (representadas por 4 classes: 4, 5, 6 e 7). A Figura 2 ilustra esse modelo e os 6 tipos de arestas presentes, sabendo que todos os tipos de arestas podem ocorrer com todas as classes de usuários. Vale ressaltar ainda que o modelo proposto permite capturar o deslocamento dos usuários entre bairros de diferentes classes socioeconômicas, independente da classe do usuário. Os tipos de arestas presentes no modelo estão relacionadas a seguir:

- laranja: deslocamento no mesmo bairro no mesmo instante de tempo;

- vermelho: deslocamento no mesmo bairro em diferentes instantes de tempo;

- azul: deslocamento entre bairros da mesma classe no mesmo instante de tempo;

- verde: deslocamento entre bairros da mesma classe em diferentes instantes de tempo;

- roxo: deslocamento entre bairros de diferentes classes no mesmo instante de tempo;

- marrom: deslocamento entre bairros de diferentes classes em diferentes instantes de tempo.

\subsubsection{Métricas de Centralidades}

Com esse modelo é possível recuperar o vértice mais central determinando o grau dos vértices. O grau geral de um vértice $v$ é o número de arestas incidentes a esse vértice, o grau de entrada é o número de arestas que apontam para $v$ e o grau de saída é o número de arestas que saem de $v$. De acordo com a Figura 2 o vértice com maior grau é o bairro 2 da classe 4 no instante t2, com grau 6 . Ainda nessa figura é possível visualizar o vértice com maior grau de entrada, que neste caso é o bairro 1 da classe 7 em t 2 com valor igual a 4. Finalizando o conceito de grau, o vértice com maior grau de saída é o bairro 2 da classe 5 em t2, também com valor igual a 4 .

Outra forma de recuperar o vértice mais central é pelo valor de intermediação (betweenness), que mede a importância dos vértices em relação ao fluxo de informações entre todos os pares de vértices, assumindo que as informações fluem principalmente 


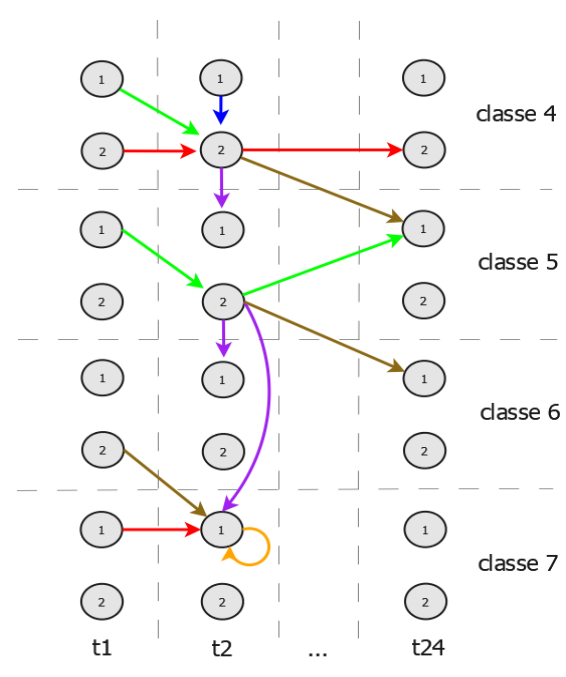

Figura 2. Representação da estrutura do grafo geral utilizando MAG

pelos menores caminhos entre eles [Newman 2010]. A intermediação de um vértice $v$ é dada pela Equação 1, onde $\sigma_{s t}$ é o número total de menores caminhos do vértice $s$ para o vértice $t$ e $\sigma_{s t}(v)$ é o número de menores caminhos que passam por $v$.

$$
g(v)=\sum_{s \neq v \neq t} \frac{\sigma_{s t}(v)}{\sigma_{s t}}
$$

\subsubsection{Subdeterminação do MAG}

A subdeterminação de um MAG (MAG Subdetermination) consiste em suprimir um ou mais aspectos do modelo, consequentemente reduzindo sua ordem. Este processo pode continuar até que o MAG seja transformado em um grafo tradicional, ou seja, com apenas um aspecto. Uma lista binária $\zeta$ indica quais aspectos do MAG original estão presentes na subdeterminação. O MAG desse trabalho foi estabelecido com os aspectos na seguinte ordem: a classe socioeconômica do indivíduo que está realizando o deslocamento, os bairros da cidade, os instantes de tempo e a classe socioeconômica do bairro. Diante disso, o bit menos significativo (mais à direita) indica a ausência ou presença do último aspecto e o bit mais significativo (mais à esquerda) indica a ausência ou presença do primeiro aspecto. Portanto, para cada $\zeta$, tem-se uma sublista única $A_{\zeta}(H)$ de aspectos, de tal modo que $p_{\zeta}=\left|A_{\zeta}(H)\right|$ é a ordem da subdeterminação $\zeta$. Além da sublista $A_{\zeta}(H)$ de aspectos, existem também as arestas subdeterminadas obtidas a partir de $\zeta$. Com base nisso, o MAG subdeterminado é obtido através da função [Wehmuth et al. 2016]:

$$
\begin{array}{r}
M_{\zeta}:(A(H), E(H)) \rightarrow\left(A_{\zeta}(H), \mathbf{E}_{\zeta}(H)\right) \\
H \mapsto\left(A_{\zeta}(H), E_{\zeta}(E(H))\right)
\end{array}
$$

Sabendo que $\mathbf{E}_{\zeta}(H)$ é o conjunto de todas as arestas subdeterminadas possíveis e considerando que duas arestas distintas $e_{1}, e_{2} \epsilon E(H)$ se diferem apenas em aspectos que não estão em $A_{\zeta}(H)$, afirma-se que $E_{\zeta}\left(e_{1}\right)=E_{\zeta}\left(e_{2}\right)$. Desta forma, $A_{\zeta}(H)$ é a sublista 
de aspectos de $H$ prescritas por $\zeta$ e $E_{\zeta}(E(H))$ é o conjunto de todas as arestas subdeterminadas de acordo com a subdeterminação $\zeta$, disto segue que $\left(A_{\zeta}(H), E_{\zeta}(E(H))\right)$ é um sub-MAG obtido de $H$ de acordo com a subdeterminação $\zeta$.

\section{Resultados}

Nesta seção são apresentados os resultados obtidos com a proposta deste trabalho.

\subsection{Informações Socioeconômicas sobre a População de Curitiba}

Primeiramente são apresentados os resultados para o processo de estratificação socioeconômica. A Tabela 2 apresenta de forma simplificada a distribuição da população de Curitiba de acordo com sua classificação socioeconômica e o número de usuários distintos de cartões transporte por classe, com o total de viagens analisadas neste estudo.

Tabela 2. Distribuição da população de acordo com a classe socioeconômica

\begin{tabular}{cccccc} 
& Curitiba & CMB & CM & CMA & CAB \\
\hline população oficial & 1.751 .907 & 97.910 & 530.683 & 602.653 & 520.661 \\
\hline$\%$ & & $5,59 \%$ & $30,29 \%$ & $34,40 \%$ & $29,72 \%$ \\
\hline usuários distintos de cartões analisados & 14.632 & 330 & 3.412 & 3.826 & 7.064 \\
\hline$\%$ & & $2,26 \%$ & $23,32 \%$ & $26,15 \%$ & $48,28 \%$ \\
\hline viagens analisadas & 721.910 & 14.966 & 163.776 & 180.425 & 362.743 \\
\hline$\%$ & & $2,07 \%$ & $22,69 \%$ & $24,99 \%$ & $50,25 \%$ \\
\hline
\end{tabular}

Ao analisar a Tabela 2 observa-se que o maior número de habitantes de Curitiba pertencem à Classe CMA. Em termos de cartão transporte analisados é a Classe CAB que se destaca, com quase 50\% dos usuários. Analisando o banco de dados para esse estudo, observa-se também que a maioria dos usuários do cartão transporte são da Classe $\mathrm{CAB}$, corroborando com as informações apresentadas na tabela.

\subsection{Grafo de Mobilidade}

\subsubsection{Grafo Geral}

Ao estabelecer o grafo geral com a utilização do MAG obteve-se uma estrutura com 4.204 vértices e 63.718 arestas direcionadas e ponderadas. A direção das arestas identifica o sentido do deslocamento, já o peso corresponde a quantidade de deslocamentos que ocorreu entre dois vértices. Ao extrair deste modelo as 20 arestas mais pesadas observou-se a predominância do bairro Centro, tanto como origem quanto como destino, ou seja, a maior quantidade de deslocamentos ocorre dentro deste bairro, compondo as 10 arestas mais pesadas do modelo e obtendo o maior peso único, 1.034 deslocamentos. O segundo bairro mais dominante é Cidade Industrial, aparecendo em 4 arestas como origem, sendo o Centro seu destino. Compondo os deslocamentos mais intensos ainda aparecem os bairros Capão Raso, Água Verde, Vista Alegre e Bairro Alto, com arestas de peso menor que 294. Este resultado é o que caracteriza também o grafo de mobilidade criado a partir da matriz de origem e destino dos usuários em nosso trabalho anterior [Santin et al. 2020]. A partir desta matriz foi criado o grafo tradicional da Figura 3, onde é possível verificar que os deslocamentos mais intensos envolvem os bairros Centro e Cidade Industrial, demonstrados pela aresta mais espessa.

No entanto, com o MAG é possível identificar a classe socioeconômica do usuário e os horários em que esses deslocamentos mais intensos ocorrem. Das 20 arestas mais 


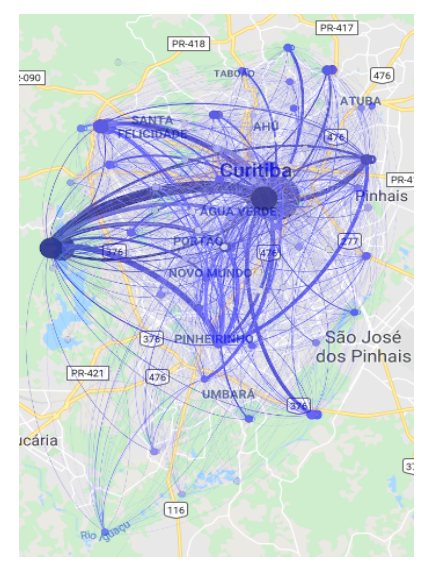

Figura 3. Visão Geral da Rede de Mobilidade de Curitiba através de um grafo tradicional (sem levar em consideração o aspecto tempo e classe socioeconômica) [Santin et al. 2020, p. 8]

pesadas, 16 são da classe CAB e 4 da classe CM. Os deslocamentos têm origem às $6 \mathrm{~h}$, $7 \mathrm{~h}$ ou $8 \mathrm{~h}$, com o retorno a partir das $12 \mathrm{~h}$. Este resultado temporal corrobora com os horários escolhidos para inferir o bairro de origem e de destino em nosso estudo anterior [Santin et al. 2020], onde foi determinado como origem o bairro mais frequente das entradas feitas até $10 \mathrm{~h}$ e como destino, o bairro mais frequente das entradas realizadas a partir deste horário.

\subsubsection{Grafo por Classes Socioeconômicas}

Para analisar a mobilidade do transporte público nos diferentes estratos socioeconômicos de Curitiba, a saber, as classes CMB, CM, CMA e CAB (as únicas observadas na cidade de acordo com o Censo de 2010), foram analisados 4 grupos de arestas, onde cada grupo corresponde aos deslocamentos dos usuários de uma classe, preservando os aspectos das 4 camadas como classe socioeconômica do bairro (4, 5, 6 e 7), os 24 instantes de tempo (cada instante corresponde a $1 \mathrm{~h}$ do dia) e os bairros da cidade.

Em cada grupo recuperou-se o vértice mais central, considerando o grau dos vértices (geral, de entrada e de saída) e o valor de intermediação. Este resultado está apresentado na Tabela 3.

\begin{tabular}{|c|c|c|c|c|}
\hline & CMB & $\mathbf{C M}$ & CMA & CAB \\
\hline Grau geral & Tatuquara-6h & Cidade Industrial-6h & Centro-17h & Centro-7h \\
\hline Grau de entrada & Pinheirinho-14h & Centro- $18 \mathrm{~h}$ & Centro-17h & Centro-18h \\
\hline Grau de saída & Tatuquara-6h & Cidade Industrial-6h & Santa Cândida-6h & Centro-7h \\
\hline Intermediação & Campo de Santana-17h & Centro- $12 \mathrm{~h}$ & Centro-17h & Centro-12h \\
\hline
\end{tabular}

É possível notar a predominância de primeiros horários da manhã (6h e 7h) ao considerar o vértice com maior grau geral e maior grau de saída em cada classe, assim como, uma grande ocorrência de horários de fim de tarde (17h e $18 \mathrm{~h})$ em vértices com maior grau de entrada, reforçando os padrões temporais encontrados em [Santin et al. 2020], 
que mostra dois picos relevantes no dia, um no início da manhã e outro no fim da tarde. Todavia, com o MAG é possível observar simultaneamente ao horário, o bairro presente nestes pontos centrais, mostrando um forte deslocamento com origem em Tatuquara para a classe CMB, Cidade Industrial para a classe CM, Santa Cândida para a classe CMA e Centro para a classe CAB.

Este resultado ainda pode exemplificar o valor de assortatividade encontrada em nosso estudo anterior [Santin et al. 2020]. Esta medida calcula a semelhança das conexões na rede com relação a um determinado atributo [Newman 2002], a classe socioeconômica foi o atributo escolhido e nosso estudo mostrou uma tendência das pessoas se deslocarem entre bairros da mesma classe. O bairro Centro pode ser tratado como uma exceção por ser a região central da cidade e, por isso, aparece como destino predominante para diferentes classes socioeconômicas de indivíduos. Já o Pinheirinho (bairro classe 5) na classe $\mathrm{CMB}$, pode não consolidar o resultado da assortatividade pela sua localização geográfica. Compondo o maior grau de entrada da classe CMB e ocorrendo às $14 \mathrm{~h}$, os resultados sugerem que o Pinheirinho é um destino intermediário ou final bastante comum por ser um bairro de fronteira com Tatuquara, que é um dos bairros mais distantes do Centro.

Os vértices com maior centralidade de intermediação são os mesmos para a classe $\mathrm{CM}$ e $\mathrm{CAB}$, sendo Centro às $12 \mathrm{~h}$. O Centro compõe o local mais importante também para a classe CMA, porém às $17 \mathrm{~h}$, o que reforça a dominância deste bairro em participar de destinos intermediários além de destinos finais, para 3 das 4 classes socioeconômicas. A presença do bairro Campo de Santana às $17 \mathrm{~h}$ para a classe CMB foi analisada mais criteriosamente com o objetivo de entender o motivo desse bairro aparecer nesta análise. Observando os deslocamentos completos que possuem este vértice como destino intermediário, foi detectado que os indivíduos realizam deslocamentos dentro deste bairro, um pela manhã e outro no fim da tarde (precisamente às $17 \mathrm{~h}$ ) porque provavelmente trabalham no mesmo bairro em que moram, e após o trabalho se deslocam para outros bairros da cidade, os quais são mais próximos da região central, como Centro Cívico, Água Verde e Bairro Alto, possivelmente para ter acesso a locais e serviços, como faculdades e universidades, não disponíveis em sua região (lembrando que Campo de Santana está localizado no extremo sul da cidade), e retornando mais tarde às suas casas.

Analisando ainda as 10 arestas mais pesadas por classe, foi possível identificar um padrão em relação aos horários de início dos deslocamentos, mostrando que com o aumento da riqueza a atividade matinal é adiada, da mesma forma que inferimos em nosso trabalho anterior [Santin et al. 2020]. Predominantemente, na classe CMB os deslocamentos se originam às $5 \mathrm{~h}$ em Tatuquara, na $\mathrm{CM}$ às $6 \mathrm{~h}$ na Cidade Industrial, na classe CMA às $7 \mathrm{~h}$ no Capão Raso e Bairro Alto e na $\mathrm{CAB}$, também ocorre às $7 \mathrm{~h}$, porém no Centro. Como é possível perceber, com o MAG pudemos enriquecer as informações de tempo, extraindo concomitantemente as informações de onde tais deslocamentos se iniciam.

\subsection{A Importância do Aspecto Socioeconômico}

Na busca de dimensionar a importância do aspecto classe socioeconômica do indivíduo, foi aplicado o conceito de subdeterminação do grafo em nosso modelo utilizando $\zeta=$ $0111_{2}$, já que o aspecto suprimido foi a classe socioeconômica do indivíduo. Com a omissão deste aspecto, o novo modelo permite a visualização dos deslocamentos entre 
classes de bairros, entre bairros e em diferentes horas do dia, sendo possível encontrar os vértices mais centrais sem considerar a classe social de quem realizou estes deslocamentos. Porém, para evitar inconsistências, os atributos dos vértices e das arestas (inclusive o peso) não são mantidos ao criar o sub-MAG e, portanto, foi necessário calcular e inserir os pesos das arestas para que o sub-MAG ficasse compatível com o MAG original.

Utilizando os mesmos artifícios aplicados no grafo da seção anterior para recuperar os vértices mais centrais de acordo com o grau do vértice e o valor de intermediação, obteve-se o Centro em todos os resultados. O vértice com maior grau ocorre às $7 \mathrm{~h}$, exatamente o mesmo da classe $\mathrm{CAB}$, isso pode ocorrer pela quantidade de viagens desta classe em nossa base, em torno de $50 \%$. Já o vértice com maior intermediação deu-se às 14h. Estes resultados denotam uma perda de detalhes importantes que são encontrados quando sabe-se a classe socioeconômica de quem realizou os deslocamentos. O Centro, por exemplo, pode ser um destino intermediário para a classe $\mathrm{CMB}$, porém não é o mais importante, este bairro sequer apareceu em uma das métricas analisadas para esta classe.

Ao analisar o grafo suprimindo a informação da classe socioeconômica do usuário é possível identificar quais bairros e horários são de maior movimentação no grafo estabelecido. Entretanto, incluindo este aspecto é possível capturar a diversidade de comportamento em cada estrato socioeconômico. Neste sentido, análises com maior detalhamento como as permitidas pela estrutura completa do MAG podem ser úteis, por exemplo, para as administrações públicas, como indicação onde as campanhas de prevenção de doenças e de vacinação atingiriam um maior público. Ainda, em tempos de necessidade de ações mais efetivas para limpeza de espaços [Prefeitura Municipal de Curitiba 2020b], essa identificação poderia ser utilizada como norteadora para a priorização dessas ações.

\section{Conclusão}

Este artigo apresenta um estudo sobre a mobilidade urbana de Curitiba utilizando dados de transporte coletivo disponibilizados por órgãos públicos da cidade. Adicionando informações sobre a classe socioeconômica dos usuários, a mobilidade foi analisada utilizando-se de um recurso multicamadas, Multi-Aspect Graph (MAG), ao invés de um grafo tradicional. Para evidenciar as vantagens de utilizar um MAG foram realizadas comparações com os resultados obtidos em nosso trabalho prévio, no qual foi criado um modelo representando os deslocamentos de origem e destino dos usuários do transporte público de Curitiba utilizando um grafo tradicional e, em seguida, foram extraídos padrões espaço-temporais considerando também subconjuntos correspondentes a estratos socioeconômicos.

O grafo de mobilidade geral caracteriza a mobilidade urbana sob 4 aspectos, permitindo a análise conjunta de tempo (de hora em hora), espaço (bairros) e classe socioeconômica do indivíduo, realizando deslocamentos entre as 4 camadas correspondentes à classe socioeconômica dos bairros. A partir deste grafo foram identificados os deslocamentos mais intensos da cidade, os quais envolvem majoritariamente o horário do início da manhã ( $6 \mathrm{~h}$ às $8 \mathrm{~h}$ ) como origem e a partir de $12 \mathrm{~h}$ como deslocamentos de retorno. Ainda nesses deslocamentos mais intensos destacam-se as classes $\mathrm{CAB}$ e $\mathrm{CM}$ e os bairros Centro e Cidade Industrial. Todas estas informações não são possíveis de serem extraídas com um único grafo tradicional, já que este considera os deslocamentos espaço-temporais de forma agrupada. 
Ao estudar o grafo por classe socioeconômica do indivíduo muitos resultados reforçaram padrões temporais encontrados em nosso estudo anterior [Santin et al. 2020], como os primeiros horários da manhã ( $6 \mathrm{~h}$ e $7 \mathrm{~h}$ ) identificando o momento em que os indivíduos estão indo trabalhar ou estudar (maior grau de saída dos vértices) e horários de fim de tarde (17h e 18h) como o retorno dos indivíduos para suas casas (maior grau de entrada dos vértices). Porém com o MAG, é possível constatar simultaneamente ao horário, os bairros presentes nestes pontos centrais de cada classe, sendo Tatuquara, Pinheirinho e Campo de Santana para a classe CMB, Cidade Industrial para CM, Santa Cândida para CMA e Centro para CM, CMA e CAB. Nestes horários e locais é, portando, detectada uma maior aglomeração de pessoas e este resultado é capaz de auxiliar as empresas de transporte público a planejar aumentos da frota nestes pontos e horários, garantindo atender toda a cidade de Curitiba, já que nesta análise pôde-se considerar o detalhamento comportamental por classe socioeconômica. Esta é uma ação que pode ser essencial no auxílio ao combate a uma pandemia como a COVID-19, ao qual estamos vivenciando e que exige a eliminação de agrupamentos de pessoas para diminuir o contágio.

O uso de uma estrutura como o MAG possibilita também analisar o grafo suprimindo um de seus aspectos (MAG Subdetermination). Em nosso modelo o aspecto omitido foi a classe socioeconômica do indivíduo, justamente para analisarmos a importância deste aspecto em nosso grafo. Nesta perspectiva foi possível determinar os vértices mais centrais do grafo sem levar em conta a classe socioeconômica do indivíduo que realizou os deslocamentos. Nesta análise obteve-se o Centro como vértice mais central em relação ao grau e ao valor de intermediação. Observa-se uma perda de detalhamento ao realizar esta análise, pois o Centro, por exemplo, nem sequer apareceu mas métricas analisadas para a classe $\mathrm{CMB}$, não demostrando ser o mais importante para ela.

Um panorama da mobilidade urbana sob aspectos espaciais e temporais, considerando os estratos socioeconômicos dos bairros e dos indivíduos que realizam os deslocamentos pode beneficiar no trabalho de diversos profissionais, como planejadores urbanos, sociólogos, engenheiros e epidemiologistas, dando direções de locais e horários de maior aglomeração, além de identificar deslocamentos estratégicos para a formulação de novas rotas de ônibus. Com o MAG existe a possibilidade de trabalhar com todos esses aspectos em um único modelo, garantindo um detalhamento das informações recuperadas e uma flexibilidade em criar modelos derivados ao surgir a necessidade de perspectivas integradas em relação a alguma particularidade, constituindo assim, algumas das vantagens sobre um modelo tradicional.

\section{Agradecimentos}

Este trabalho foi parcialmente apoiado pelo projeto CNPq-UrbComp (403260/2016-7), CNPq (PIBIC 128796/2019-7), CAPES (código de financiamento 001) e projeto GoodWeb (\#2018/23011-1 FAPESP).

\section{Referências}

Araujo, M., Melo de Oliveira, J., Jesus, M., Sa, N., Santos, P., and Lima, T. (2011). Collective public transportation: Discussing accessibility, mobility and quality of life. Psicologia Sociedade, 23:574-582.

El Mahrsi, M. K., Côme, E., Oukhellou, L., and Verleysen, M. (2017). Clustering smart card data for urban mobility analysis. IEEE Transactions on Intelligent Transportation Systems, 18(3):712-728.

Governo de São Paulo (2019). Governo de sp implementa nova política de mobilidade metropolitana. https://www.saopaulo.sp.gov.br/spnoticias/governo-de-sp-implementa-nova-politica-de-mobilidade-metropolitana/. [Online; acessado em 15-Abril-2020]. 
Guttman, A. (1984). R-trees: A dynamic index structure for spatial searching. SIGMOD Rec., 14(2):47-57.

Herek, T. A., Munaretto, A., Fonseca, M. S. P., and Monego, H. I. D. (2018). Análise da capacidade de entrega de uma rede oportunista no transporte público urbano. In Anais do II Workshop de Computação Urbana, Porto Alegre, Brasil. SBC.

Instituto Brasileiro de Geografia e Estatística - IBGE (2010). Censo 2010. https://censo2010.ibge.gov.br. [Online; acessado em 07-Novembro-2019].

Kivela, M., Arenas, A., Barthelemy, M., Gleeson, J. P., Moreno, Y., and Porter, M. A. (2014). Multilayer networks. Journal of Complex Networks, 2(3):203-271.

Lotero, L., Hurtado, R. G., Floría, L. M., and Gómez-Gardeñes, J. (2016). Rich do not rise early: spatio-temporal patterns in the mobility networks of different socio-economic classes. Royal Society Open Science, 3(10):150654.

Marques-Neto, H. T., Xavier, F. H. Z., Xavier, W. Z., Malab, C. H. S., Ziviani, A., Silveira, L. M., and Almeida, J. M. (2018). Understanding human mobility and workload dynamics due to different large-scale events using mobile phone data. Journal of Network Systems Management, 26(4):1079 - 1100.

Martins, K. S. and Cunha, F. D. (2018). Explorando dados urbanos: um estudo usando viagens de táxi da cidade de são francisco. In Anais do II Workshop de Computação Urbana, Porto Alegre, Brasil. SBC.

Morais, M. and Camargo, R. (2019). A framework for scalable data analysis and model aggregation for public bus systems. In Anais do III Workshop de Computação Urbana, pages 83-96, Porto Alegre, Brasil. SBC.

Newman, M. (2010). Networks: An Introduction. Oxford University Press.

Newman, M. E. J. (2002). Assortative mixing in networks. Phys. Rev. Lett., 89:208701.

Ninis, A. B., Paes de Barros, R., Grosner, D., Franco, S., Rosalém, A., Bugarin, Karina Sayuri Sataka, G., and Jorge, J. (2012). SAE Relatorio de definicao da classe media.

Nunes, I. O., Celes, C., de Melo, P. O. S. V., and Loureiro, A. A. F. (2017). Modelo de mobilidade para encontros de grupos. In Anais do I Workshop de Computação Urbana, Porto Alegre, Brasil. SBC.

Prefeitura Municipal de Curitiba (2020a). Curitiba e mit seguem estudos em mobilidade e inovação. https://www.curitiba.pr.gov.br/noticias/curitiba-e-mit-seguem-estudos-em-mobilidade-e-inovacao/55548. [Online; acessado
[ em 15-Abril-2020].

Prefeitura Municipal de Curitiba (2020b). Prefeitura reforça limpeza dos 22 terminais da cidade. https://www.curitiba.pr.gov.br/noticias/prefeitura-reforca-limpeza-dos-22-terminais-da-cidade/55469. [Online; acessado em 09-Abril-2020].

Prefeitura Municipal de Porto Alegre (2019). Prefeitura apresenta situação das obras de mobilidade da capital. https://prefeitura.poa.br/smim/noticias/prefeitura-apresenta-situacao-das-obras-de-mobilidade-da-capital. [Online; acessado em 15-Abril-2020].

Rodrigues, D. O., Boukerche, A., Silva, T. H., Loureiro, A. A., and Villas, L. A. (2018a). Combining taxi and social media data to explore urban mobility issues. Computer Communications, 132:111-125.

Rodrigues, F., Xavier, C. R., Evsukoff, A. G., Ziviani, A., and da F. Vieira, V. (2018b). Análise de mobilidade urbana em diferentes estratos sociais a partir de uma rede de telefonia móvel. In Anais do II Workshop de Computação Urbana, Porto Alegre, Brasil. SBC.

Santin, P., Gubert, F. R., Fonseca, M., Munaretto, A., and Silva, T. H. (2020). Enriquecendo modelos de mobilidade urbana com padrões espaço-temporais e estratos socioeconômicos. In Simpósio Brasileiro de Redes de Computadores e Sistemas Distribuídos, Rio de Janeiro, Brasil.

Santin, P., Munaretto, A., Fonseca, M., and Silva, T. H. (2019). Integração de dados públicos de saúde e transporte: Caracterização para modelagem multicamadas. In III Workshop de Computação Urbana, Gramado, Brasil.

Silva, T. H., Viana, A. C., Benevenuto, F., Villas, L., Salles, J., Loureiro, A., and Quercia, D. (2019). Urban computing leveraging location-based social network data: A survey. ACM Comput. Surv., 52(1):17:1-17:39.

Taniguchi, G. and Duarte, F. (2012). Personal smart cards: From transportation to a city smart card-the database integration of public services in Curitiba. In City Competitiveness and Improving Urban Subsystems: Technologies and Applications, pages $217-232$. IGI Global.

Urbanização de Curitiba S/A - URBS (2018). URBS em números. https://www.urbs.curitiba.pr.gov.br/institucional/urbs-em-numeros. [Online; acessado em 07-Novembro-2019].

Wehmuth, K., Fleury, E., and Ziviani, A. (2016). On multiaspect graphs. Theoretical Computer Science, 651:50-61.

Xu, Y., Belyi, A., Bojic, I., and Ratti, C. (2018). Human mobility and socioeconomic status: Analysis of Singapore and Boston. Computers, Environment and Urban Systems, 72:51 - 67.

Zhang, X., Xu, Y., Tu, W., and Ratti, C. (2018). Do different datasets tell the same story about urban mobility - A comparative study of public transit and taxi usage. Journal of Transport Geography, 70:78-90.

Átila M. Silva Jr, Marques-Neto, H. T., and Gonçalves, J. M. A. (2018). Custos e benefícios de viagens compartilhadas em um sistema de transporte multimodal. In Anais do II Workshop de Computação Urbana, Porto Alegre, Brasil. SBC. 